\title{
More Forbidden Minors for Wye-Delta-Wye Reducibility
}

\author{
Yaming $\mathrm{Yu}$ \\ Department of Statistics \\ University of California \\ Irvine, CA 92697, USA \\ yamingy@uci.edu
}

Submitted: Mar 5, 2005; Accepted: Jan 18, 2006; Published: Jan 25, 2006

Mathematics Subject Classifications: 05C75, 05C83

\begin{abstract}
A graph is $Y \Delta Y$ reducible if it can be reduced to isolated vertices by a sequence of series-parallel reductions and $Y \Delta Y$ transformations. It is still an open problem to characterize $Y \Delta Y$ reducible graphs in terms of a finite set of forbidden minors. We obtain a characterization of such forbidden minors that can be written as clique $k$-sums for $k=1,2,3$. As a result we show constructively that the total number of forbidden minors is more than 68 billion up to isomorphism.
\end{abstract}

\section{Introduction}

We follow the terminology of Archdeacon et al. [1]. The graphs under consideration are finite, undirected, but may have loops or multiple edges. The series-parallel reductions are defined by the following four operations:

- Loop reduction: Delete a loop.

- Degree-one reduction: Delete a degree-one vertex and its incident edge.

- Series reduction: Delete a degree-two vertex $y$ and its two incident edges $x y$ and $y z$, and add the new edge $x z$.

- Parallel reduction: Delete one of a pair of parallel edges.

The class of graphs that can be reduced to isolated vertices by these four reductions is called series-parallel reducible. (Disconnected graphs are allowed for convenience.) The $Y \Delta$ and $\Delta Y$ transformations are defined as follows: 
- $Y \Delta$ transformation: Delete a degree-three vertex $w$ and its three incident edges $w x$, $w y$ and $w z$, and add three edges $x y, y z$ and $x z$.

- $\Delta Y$ transformation: Delete the three edges of a triangle (delta) xyz, and add a new vertex $w$ and three new edges $w x, w y$ and $w z$.

Two graphs that can be obtained from each other by a sequence of $Y \Delta Y$ transformations are called $Y \Delta Y$ equivalent. The class of graphs that can be reduced to isolated vertices by the above six reductions/transformations is called $Y \Delta Y$ reducible.

A graph $H$ is a minor of a graph $G$, if $H$ can be obtained from $G$ by a sequence of edge deletions, edge contractions, and deletions of isolated vertices. A class of graphs is minor closed if for graph $G$ in the class, any minor $H$ of $G$ is also in the class. According to the deep results of Robertson and Seymour [7], a minor closed graph family is characterized by a finite set of forbidden minors, i.e., graphs that are not in the family but every minor is in the family.

Truemper [8] shows that the class of $Y \Delta Y$ reducible graphs is minor closed. The forbidden minor characterization for this class of graphs, however, is still open. According to Epifanov [2] (see also [4, 3]), all planar graphs are $Y \Delta Y$ reducible. There are 57587 known graphs from the literature that belong to the finite set of minor-minimal $Y \Delta Y$ irreducible graphs. These graphs fit in four $Y \Delta Y$ equivalent families, one of which has 57578 members. Yu [10] charters the 57578-member family with the aid of a computer.

The main result of the current paper is to present additional families of graphs that are forbidden minors for $Y \Delta Y$ reducibility. Specifically, a total of 68897913659 graphs, including the known 57587, are shown to be minor-minimal. These graphs fit in $20 Y \Delta Y$ equivalent families. The result is obtained by a combination of detailed analysis and computer confirmation.

The concept of terminal $Y \Delta Y$ reducibility is instrumental to the development of this paper. For a graph $G$, let $T \subset G$ be a set of distinguished vertices, called terminals. $G$ is terminal $Y \Delta Y$ reducible (or T-reducible) if all non-terminal vertices can be reduced to isolated vertices by performing series-parallel reductions and $Y \Delta Y$ transformations without ever deleting any vertex in $T$. A minor $H$ of a graph $G$ with terminals is called a terminal minor (or T-minor) of $G$ if $H$ can be obtained from $G$ by edge deletion, edge contraction and deletion of isolated vertices, without deleting any of the terminals, or contracting any edges between terminals. These two concepts are introduced in Archdeacon et al. [1].

Two graphs $H$ and $H^{\prime}$ with terminals are said to be T-isomorphic if there is a bijection between the vertices of $H$ and $H^{\prime}$ that maps terminals to terminals, non-terminals to nonterminals, and also preserves adjacency. If $H$ is T-isomorphic to a T-minor of $G$, we say $G$ contains an $H$ T-minor.

Connectivity considerations are important for this work. For a graph $G$, a separation is a pair of subgraphs $\left(G_{1}, G_{2}\right)$ such that $G_{1} \cup G_{2}=G$ and $E\left(G_{1}\right) \cap E\left(G_{2}\right)=\emptyset$. The order of the separation is $\left|V\left(G_{1}\right) \cap V\left(G_{2}\right)\right|$. We call $C=V\left(G_{1}\right) \cap V\left(G_{2}\right)$ the cut set. $G$ is $k$-connected if $|V(G)|>k$ and there is no (nontrivial) separation of order less than $k$. $G$ is internally 4 -connected if $G$ is 3 -connected and for every separation $\left(G_{1}, G_{2}\right)$ of order 
three, either $\left|E\left(G_{1}\right)\right| \leq 3$ or $\left|E\left(G_{2}\right)\right| \leq 3$. In this paper, we call a separation essential, if each of $G_{1}-C$ and $G_{2}-C$ has at least two vertices.

The rest of the paper is organized as follows. We begin with some preliminary results on the connectivity properties of the forbidden minors. In particular, Section 2 shows that the known 57587 forbidden minors are internally 4-connected. Section 3 then naturally explores the possibility of forbidden minors with lower connectivity, which leads to a characterization of such forbidden minors in terms of clique sums of very special graphs called T-critical graphs. Finally it is shown that forbidden minors that are not internally 4-connected exist in large numbers.

\section{Connectivity and $Y \Delta Y$ Reducibility}

We have the following proposition, which follows, for example, from Theorem 2.1 of Archdeacon et al. [1] (the Minor Theorem).

Proposition 2.1 Let $G$ be minor-minimal (terminal) $Y \Delta Y$ irreducible. Then $G$ does not have loops, parallel edges, or non-terminal vertices with degree one or two. Furthermore, graphs obtained from $G$ by $Y \Delta Y$ transformations are also minor-minimal.

We now explore connectivity properties of $G$ under $Y \Delta Y$ transformations. If $G$ is minor-minimal $Y \Delta Y$ irreducible, observe that for $k=1,2$ every $k$-separation of $G$ is essential. If there is a 3 -separation $\left(G_{1}, G_{2}\right)$ with cut set $C$ and either $G_{1}-C$ or $G_{2}-C$ has just a single vertex, then there are no edges between vertices in $C$. It follows that $G$ being internally 4-connected is equivalent to having no essential separation of order less than four. We also have

Proposition 2.2 Let $G$ be minor-minimal $Y \Delta Y$ irreducible. If $G$ has an essential separation of order at most three, then the minimum order of essential separations of $G$ does not change after $Y \Delta Y$ transformations.

Note: this is implied by the main theorem in Section 3. Below is a direct proof.

Proof. Only need to show that the minimum order of the essential separations does not increase after applying $Y \Delta$ and $\Delta Y$ transformations. This is obvious if a $\Delta Y$ transformation is applied to $G$, since any essential cut of $G$ is still an essential cut after the transformation. If a $Y \Delta$ transformation is applied, let the center of wye be $a$, with three adjacent vertices $b, c, d$. Let $\left(G_{1}, G_{2}\right)$ be an essential separation of $G$ with minimal order. Denote the cut set $C$ and consider two cases:

1. $a \in C$. Then $a$ must be adjacent to at least one vertex in each of $G_{1}-C$ and $G_{2}-C$. Since $a$ has degree three, without loss of generality assume $b \in G_{1}-C$ and $c, d \in G_{2}$. Consider the new cut set $C^{\prime}$ (for the graph after the transformation) obtained from $C$ by substituting $b$ for $a$. $C^{\prime}$ necessarily has the same order as $C$. If the new cut is not essential, $G_{1}-C$ has to be reduced to a single vertex $e$. Then in $G, b$ is adjacent to $a, e$, and at least one of the other vertices in $C$, otherwise a series reduction is applicable. Since $e$ has degree at least three and is not adjacent to $a$, there must be three vertices in $C$, and two 
of them plus $b$ are adjacent to $e$. It is clear that if we apply a $Y \Delta$ transformation using $e$ as the center of wye, a parallel edge appears, which is a contradiction.

2. $a$ is in $G_{1}-C$ or $G_{2}-C$. Assume $a \in G_{1}-C$ to be exact. In this case $C$ is still a cut set of the same order for the transformed graph. We show that the cut is still essential. Otherwise, besides $a, G_{1}-C$ has exactly one vertex, denoted $e$. Thus $a$ is adjacent to at least two vertices in $C$. After the $Y \Delta$ transformation an edge appears between these two vertices. Since $e$ is the only vertex left in $G_{1}-C$ after the transformation, $e$ has to be adjacent to all three vertices in $C$. We get the same contradiction as before.

Proposition 2.3 The known 57587 forbidden minors do not have essential separations of order less than four.

Proof. These 57587 graphs fit in four $Y \Delta Y$ equivalent families. According to the preceding proposition, we only need to show that there are no such separations for four representatives, one for each family. This is done by inspection.

Representatives of the four internally 4-connected families are depicted in Figure 1. We call a graph $G$ apex if $G-v$ is planar for some vertex $v$. Apex graphs are depicted by first drawing $G-v$, then marking the vertices adjacent to $v$ by unfilled circles. The graphs $G_{a}, G_{b}$ are apex and are drawn with this style.

$Y \Delta Y$ equivalent companions of $K_{6}$ form the seven graphs of the Petersen family, because the Petersen graph is one of them. Graph $K_{5,5}-M$ where $M$ is a perfect matching is mentioned in Archdeacon et al. [1]. Existence of minor-minimal $Y \Delta Y$ irreducible apex graphs is mentioned in Truemper [9]. $G_{a}, G_{b}$ are shown to be minor-minimal by $\mathrm{Yu}[10]$. $\left(G_{a}\right.$ is labeled as $G_{0}$ in $\mathrm{Yu}$ [10].) $G_{a}$ belongs to a $Y \Delta Y$ equivalent family of 57578 graphs. In contrast, no $Y \Delta$ or $\Delta Y$ transformation can be applied to $G_{b}$ and $K_{5,5}-M$.

\section{Forbidden Minors of Lower Connectivity}

A natural question is whether there are forbidden minors that have essential separations of orders at most three. This section gives a characterization of such forbidden minors.

Let $H$ be a simple graph with $k$ terminals. Denote by $H+\Delta(H-\Delta)$ the simple graph obtained from $H$ by adding (deleting) all possible edges between terminals. This notation is convenient, and is used whenever terminals are clearly defined. Define $H$ to be $T$-critical if

1. $H$ is not T-reducible.

2. Every T-minor of $H$ is T-reducible.

3. $H+\Delta$ is $Y \Delta Y$ reducible if terminals are not respected.

4. Each terminal is adjacent to at least one non-terminal vertex.

We now state the main theorem. 
Figure 1: Representatives of four minor-minimal $Y \Delta Y$ irreducible families.

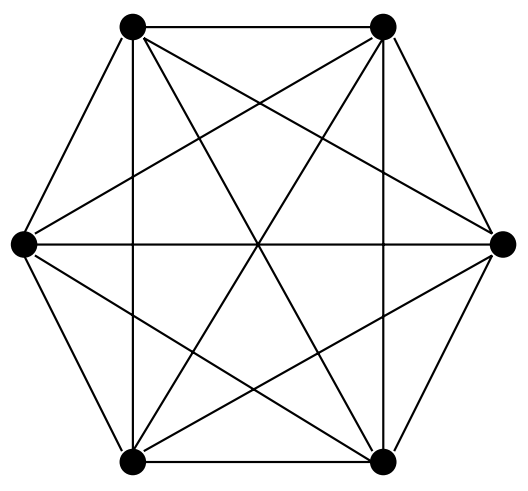

$\mathbf{K}_{6}$

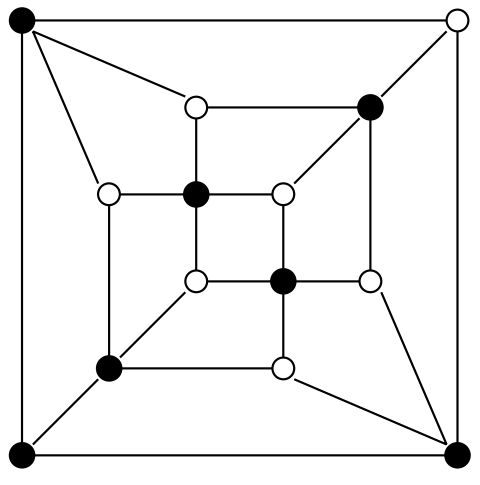

$\mathbf{G}_{\mathbf{a}}$

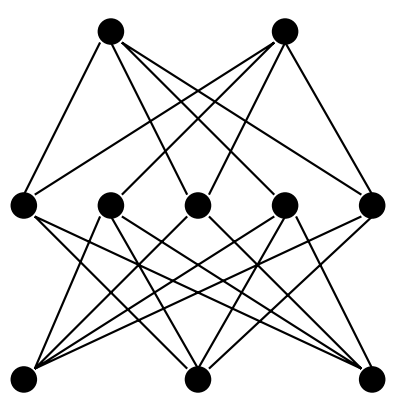

$\mathbf{K}_{5,5}-\mathbf{M}$

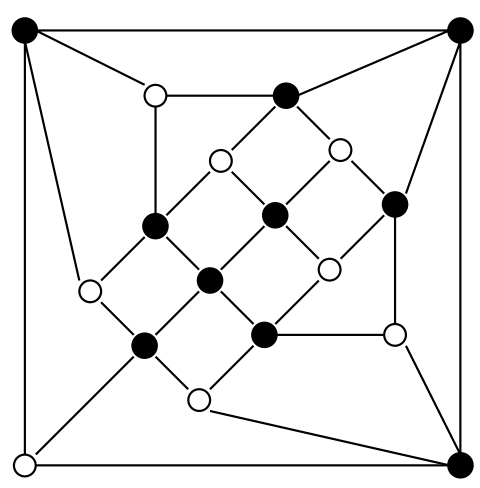

$\mathbf{G}_{\mathbf{b}}$ 
Theorem 3.1 Let $G$ be a graph whose minimum order of essential cuts is $k \leq 3$. Let $\left(G_{1}, G_{2}\right)$ be such a cut. Then $G$ is minor-minimal $Y \Delta Y$ irreducible if and only if each of $G_{i}$ is T-critical, each with the vertices in the cut set as terminals. Furthermore, in such a case, the minimal essential cut set is unique.

Proof of the theorem is presented in a series of lemmas below. We first explore simple properties of T-critical graphs.

Lemma 3.1 Let $H$ be $T$-critical and let $T$ be the set of terminals. Suppose $k=|T| \leq 3$, then

1. There are no edges between terminals.

2. $H-T$ is connected.

3. Each terminal has degree at least two.

4. If $H^{\prime}$ is obtained by applying a single $Y \Delta$ or $\Delta Y$ transformation to $H$, respecting the terminals, then $H^{\prime}$ is T-critical.

Proof. 1. If there are edges between terminals, then $H-\Delta$ is a T-minor of $H$ and is T-reducible. Furthermore, the reductions/transformations on $H-\Delta$ can be applied to $H$ correspondingly. These reductions/transformations reduce $H$ entirely, which is a contradiction.

2. If $H-T$ is disconnected, then $H+\Delta$ has a $k$-separation $\left(H_{1}, H_{2}\right)$ with $T=$ $V\left(H_{1}\right) \cap V\left(H_{2}\right)$. Therefore $H_{1}-\Delta$ and $H_{2}-\Delta$ are both T-minors of $H$, and are Treducible. $H$ is T-reducible correspondingly, a contradiction. Note, since terminals are not isolated, $H-T$ being connected implies $H$ being connected.

3. Suppose a terminal denoted $a$ has degree one and let $b$ be its only adjacent nonterminal vertex. Form set $T^{\prime}$ by substituting $b$ for $a$ in $T$. Then $H-a$ with terminals in $T^{\prime}$ is T-isomorphic to a T-minor of $H$ (with terminals in $T$ ). Hence $H-a$ is T-reducible to $T^{\prime}$ and $H$ is T-reducible after applying these reductions and a final series reduction or $Y \Delta$ transformation. This is again a contradiction.

4. The fact that $H^{\prime}$ is simple and T-minor minimal $Y \Delta Y$ irreducible follows from Proposition 2.1. Since there are no edges between terminals in $H$, the $Y \Delta$ or $\Delta Y$ transformation from $H$ to $H^{\prime}$ can be applied to $H+\Delta$ to obtain $H^{\prime}+\Delta$ (possibly resulting in a parallel edge). Hence $H^{\prime}+\Delta$ is reducible without respecting terminals. Since each terminal of $H$ is adjacent to at least two non-terminal vertices, obviously each terminal in $H^{\prime}$ is adjacent to a non-terminal vertex. By definition $H^{\prime}$ is also T-critical.

The following proposition is conveniently used in later development.

Proposition 3.1 Let $G$ be a simple $k$-connected graph, $k \leq 3$, with a $k$-separation $\left(G_{1}, G_{2}\right)$. Denote the cut set by $C$. Form $G_{i}^{\prime}, i=1,2$, from $G_{i}$ by adding all edges between vertices in $C$. Then $G_{1}^{\prime}$ is $k$-connected, and can be obtained from $G$ by a sequence of edge deletions/contractions and (possibly) a $Y \Delta$ transformation that only involve edges in $G_{2}$. 
Proof. We may assume $k \geq 2$. If $G_{1}^{\prime}$ is not $k$-connected, then there is a separation of order at most $k-1$; it is easy to show that such a separation is also a separation of $G$ itself, which is a contradiction. We may assume $G_{2}-C$ is connected. It is clear that every vertex in $C$ is adjacent to a vertex in $G_{2}-C$, otherwise a separation of lower order for $G$ results. Contract all edges in $G_{2}-C$, delete the resulting parallel edges, and apply an additional edge contraction or $Y \Delta$ transformation, we get $G_{1}^{\prime}$ from $G$.

Proposition 3.2 Under the same setting as in Proposition 3.1, suppose in addition $G$ has $k$ terminals, all contained in $G_{1}$. Declare the cut set $C$ to be the set of terminals for $G_{2}$. Assume $G$ is not T-reducible. Then both $G_{1}^{\prime}$ and $G_{2}+\Delta$ are $k$-connected. Either $G_{1}^{\prime}$ or $G_{2}$ is T-irreducible. In addition, $G_{2}$ is T-isomorphic to a T-minor of $G$.

Proof. $k$-connectivity of $G_{1}^{\prime}$ and $G_{2}+\Delta$ follows by Proposition 3.1. If both $G_{1}^{\prime}$ and $G_{2}$ are T-reducible, then $G$ is T-reducible by first applying the reductions that reduce $G_{2}$ then those that reduce $G_{1}^{\prime}$. If $C$ contains only terminal vertices of $G$, then $G_{2}$ is a T-minor of $G$. If $l>0$ vertices in $C$ are non-terminal, let $G_{0}$ be the graph $G$ after deleting the $k-l$ terminals in $C$. $G_{0}$ is necessarily $l$-connected. By Menger's Theorem, there are vertex disjoint paths in $G_{0}$ connecting the remaining $l$ terminals of $G$ with the non-terminal vertices in $C$. These paths necessarily belong to $G_{1}$. By contracting edges along these paths, it is easy to see that $G$ contains a minor T-isomorphic to $G_{2}$ (with $C$ as the set of terminals).

Lemma 3.2 If $H$ is T-critical with $k \leq 3$ terminals, then $H+\Delta$ has no essential separation of order $l \leq k$.

Proof. Assume $k \geq 2$. Assume the contrary and let $\left(H_{1}, H_{2}\right)$ be an essential separation of $H+\Delta$ of order $l$, where $H_{1}$ contains the terminals and the cut set is $C$. Assume $l$ is the minimum order of such separations. Because $H$ is T-critical, any separation of $H+\Delta$ of order less than 3 is essential, hence $H+\Delta$ is $l$-connected. (Otherwise it has a separation of order less than $l$, which is necessarily essential, a contradiction to the choice of $l$.)

We only prove the case $l=k$, for the general argument is similar. Following Proposition 3.2, $\mathrm{H}_{2}-\Delta$ (with vertices of $C$ as terminals) is a T-minor of $H$. By T-criticality, $\mathrm{H}_{2}$ is T-reducible. Since $\mathrm{H}_{2}-\mathrm{C}$ has at least two vertices, it is easy to show that $\left|E\left(H_{2}\right)\right|$ strictly decreases after these reductions/transformations. Moreover, these reductions/transformations can be performed accordingly on $H$ and decrease $|E(H)|$, which contradicts T-criticality.

Corollary 3.1 Let $H$ be $k$-terminal critical. Then $H+\Delta$ is 3-connected if $k=2$, and internally 4 -connected if $k=3$.

Proof. If $k=2$, then any 2-separation of $H+\Delta$ has to be essential, thus no such separation exists. Suppose $k=3$, and there is a 3 -separation $\left(H_{1}, H_{2}\right)$, with cut set $C$, s.t. $\left|E\left(H_{1}\right)\right|>3$ and $\left|E\left(H_{2}\right)\right|>3$. By Lemma 3.2 , one of $H_{1}-C, H_{2}-C$, say $H_{1}-C$, is a singleton, denoted $y$. Since $\left|E\left(H_{1}\right)\right|>3$, there is an edge $a b$ between two vertices in $C$. 
Perform a $Y \Delta$ transformation on $H$ using $y$ (obviously not a terminal) as the wye, we get parallel edges if one of $a, b$ is not a terminal, and an edge between terminals otherwise; both contradict T-criticality.

Corollary 3.2 A k-terminal critical graph $H$ with $k \leq 3$ is 2-connected.

Proof. We only prove the case $k=3$, because $k \leq 2$ is similar but easier. Assume $H$ has a 1-separation $\left(H_{1}, H_{2}\right)$ with cutpoint $c$. Notice that by T-criticality $c$ is an essential cut. If $c$ is a terminal, let $a$ be another terminal, then $H^{\prime}=H-\{c, a\}$ is disconnected, because $H_{1}-\{c\}$ and $H_{2}-\{c\}$ each has at least two vertices. But $H^{\prime}$ is the same as deleting $\{c, a\}$ from $H+\Delta$, and $H+\Delta$ should be 3 -connected, a contradiction. Now assume $c$ is not a terminal. If one of $H_{1}, H_{2}$ has all three terminals, then deleting $c$ disconnects $H+\Delta$; if $H_{2}$ has two terminals and $H_{1}$ has $a$, the third one, then deleting $\{c, a\}$ disconnects $H+\Delta$, again a contradiction.

Lemma 3.3 Suppose vertex-disjoint graphs $G_{1}$ and $G_{2}$ are T-critical, each with $k \leq 3$ terminals. Let there be a bijection $f$ between the two sets of terminals. Form $G$ from $G_{1}$ and $G_{2}$ by identifying each terminal of $G_{1}$ with its image under $f$. Denote by $C$ the set of $k$ merged terminal vertices. Then $G$ is $k$-connected and minor-minimal $Y \Delta Y$ irreducible.

Proof. To show that $G$ is $k$-connected we may assume $k=3$ since $k=1,2$ are easy. Suppose $G$ has a cut $\{a, b\}$ of order two. If $a, b$ belong to one of $G_{1}, G_{2}$ (say $G_{1}$ ), then $\{a, b\}$ is a separation of $G_{1}+\Delta$, a contradiction. Suppose $a \in G_{1}-C$ and $b \in G_{2}-C$. Choose $c \in C$ and $d \in G$ such that $c, d$ lie in different components of $G-\{a, b\}$. We may assume $d \in G_{1}$. Then it is easy to see that $a$ is a cutpoint for $G_{1}$, another contradiction.

To show that $G$ is not $Y \Delta Y$ reducible, notice that by T-criticality of $G_{i}, i=1,2$, there are no edges between vertices of $C$ in $G$, and each vertex in $C$ has degree at least four (at least two in each of $G_{i}$ ). No series-parallel reductions are possible for $G$, otherwise they can be applied to one of $G_{i}$, terminals respected. Hence the only possible transformations on $G$ are $Y \Delta$ and $\Delta Y$ transformations. The vertices in $C$ cannot be eliminated by a $Y \Delta$ transformation. Therefore, the $Y \Delta$ or $\Delta Y$ transformation on $G$ corresponds to the transformation on one of $G_{i}$, terminals respected. Since $Y \Delta Y$ equivalent companions of T-critical graphs are also T-critical, all the above still hold for $G$ after the $Y \Delta$ or $\Delta Y$ transformation. It follows that $G$ is not $Y \Delta Y$ reducible.

To show that every minor of $G$ is reducible. Let $G^{\prime}$ be obtained from $G$ by contracting/deleting an edge $a b$. Without loss of generality suppose $a b$ is in $G_{1}$ and let $G_{1}^{\prime}$ be the corresponding T-minor of $G_{1}$ after contracting/deleting $a b$. According to T-criticality, $G_{1}^{\prime}$ is T-reducible. Furthermore, these reductions apply to $G^{\prime}$ correspondingly and $G^{\prime}$ after these transformations is a minor of $G_{2}+\Delta . G_{2}+\Delta$ is further reducible by definition, hence $G^{\prime}$ as a whole is $Y \Delta Y$ reducible.

Note: $G$ is the clique sum of $G_{1}+\Delta$ and $G_{2}+\Delta$. We stress that there are no edges between vertices of $C$.

Lemma 3.4 Let $H$ be a graph with $k \leq 3$ terminals. Suppose $H+\Delta$ is $k$-connected and $Y \Delta Y$ reducible but $H$ is not $T$-reducible. If $H$ is not T-critical, then there is a sequence 
of edge deletions/contractions, and $Y \Delta$ transformations (terminals respected) that reduce $H$ to be T-critical.

Proof. We may assume $k \geq 2$. Let $H$ be a counterexample with the property that any counterexample either has more vertices than $H$, or has the same number of vertices but at least as many edges. If $H+\Delta$ has a separation of order $k$, then by Propositions 3.1 and 3.2 we can obtain a $k$-connected graph $H^{\prime}$ from $H+\Delta$ by a sequence of edge deletions/contractions and $Y \Delta$ transformations (terminals respected) such that $H^{\prime}$ is still T-irreducible and $\left|V\left(H^{\prime}\right)\right|<|V(H)|$, which contradicts the choice of $H$. Assume $H+\Delta$ has no separation of order $k$. Clearly there are no edges between terminals in $H$. Since $H$ is not T-critical, there is an edge $a b$ of $H$ such that the graph $H^{\prime}$ formed from $H$ by deleting/contracting $a b$ is still T-irreducible. $H^{\prime}+\Delta$ is obtained from $H+\Delta$ by the corresponding deletion/contraction. Because $H+\Delta$ has no $k$-separation, $H^{\prime}+\Delta$ is $k$-connected. Hence $H^{\prime}$ is a smaller counterexample.

Lemma 3.5 Suppose $G$ is minor-minimal $Y \Delta Y$ irreducible and suppose $\left(G_{1}, G_{2}\right)$ is an essential separation of minimal order $k \leq 3$. Then the cut set is unique and each $G_{i}, i=$ 1,2 , is T-critical with vertices in the cut as terminals.

Proof. Denote the cut set by $C$ and declare $C$ to be the terminals for $G_{i}, i=1,2$. By Proposition 3.1, $G_{i}+\Delta$ is $k$-connected and can be obtained by a sequence of edge deletions/contractions, and $Y \Delta$ transformations from $G$. Because the separation is essential, the number of edges of $G$ decreases in this process, hence at least one edge is contracted/deleted. Thus $G_{i}+\Delta$ is necessarily $Y \Delta Y$ reducible without respecting the terminals. Moreover $G_{1}$ is not T-reducible. Otherwise $G$ can be reduced to a minor of $G_{2}+\Delta$ and can then be reduced entirely, which is a contradiction. By the preceding lemma, if one of $G_{i}$ itself is not T-critical, there is a sequence of edge contractions/deletions, and $Y \Delta$ transformations that respect terminals and reduce $G_{i}$ to a T-critical graph $G_{i}^{\prime}$. Correspondingly, $G$ can be reduced by these reductions/transformations to the clique sum of $G_{1}^{\prime}+\Delta$ and $G_{2}^{\prime}+\Delta$. Since such clique sums are $Y \Delta Y$ irreducible, this contradicts the minor minimality of $G$.

To prove the uniqueness of the cut, we may assume $k \geq 2$ since $k=1$ is easy. Suppose there is another cut set $C^{\prime}$ of the same order but not identical to $C$. If all vertices of $C^{\prime}$ lie in one of $G_{1}$ or $G_{2}$ (say $G_{1}$ ), then $C^{\prime}$ is a $k$-separation of $G_{1}+\Delta$, denoted $\left(G_{11}, G_{12}\right)$. Assume $C \subset G_{11}$, and choose $c \in C$ but $c \notin C^{\prime}$. Since $c$ is adjacent to at least two vertices $d, e$ in $G_{1}-C$, we have $c, d, e \in G_{11}$. Among the $k+2$ vertices of $C \cup\{d, e\}$, at most $k$ belong to $C^{\prime}$, hence $\left|G_{11}-C^{\prime}\right| \geq 2$. Notice that $\left|G_{12}-C^{\prime}\right| \geq 2$ because $C^{\prime}$ is an essential separation of $G$ itself. It follows that $\left(G_{11}, G_{12}\right)$ is an essential separation for $G_{1}+\Delta$, which contradicts Lemma 3.2. Suppose some of the vertices of $C^{\prime}$ lie in $G_{1}-C$ and some in $G_{2}-C$. Without loss of generality assume $G_{1}-C$ contains exactly one vertex of $C^{\prime}$ denoted $a$. We may assume in addition that there are two vertices $u, v$ in $C$ such that there are no paths connecting them in $G-C^{\prime}$. If $k=2$, it follows that every path that connects $u, v$ in $G_{1}$ contains vertex $a$. Hence $G_{1}$ has a cutpoint, which contradicts Corollary 3.2. A similar contradiction results when $k=3$ and $C \cap C^{\prime}=\emptyset$. Now assume 
$k=3$ and $\left|C \cap C^{\prime}\right|=1$, with $x \in C \cap C^{\prime}$. It is clear that every path connecting $u, v$ in $G_{1}$ has to contain either $a$ or $x$, so $\{a, x\}$ separates $G_{1}$. It follows that $\{a, x, u\}$ separates $G_{1}+\Delta$. Denote the separation $\left(G_{11}, G_{12}\right)$ and assume $v \in G_{11}-\{a, x, u\}$. By Lemma 3.2 , such a separation should not be essential. Since $v$ is adjacent to at least two vertices in $G_{1}-C,\left|G_{11}-\{a, x, u\}\right| \geq 2$. Hence $G_{12}-\{a, x, u\}$ is a singleton, denoted $y$. It is only possible for $y$ to be adjacent to $u, a, x$ in $G$, hence $y$ is adjacent to all three. By T-criticality of $G_{1}, u$ has to be adjacent to at least two vertices in $G_{1}-C$, so $u$ has to be adjacent to both $a$ and $y$. If we apply a $Y \Delta$ transformation to eliminate $y$, a parallel edge au results, which is a contradiction.

\section{Construction of T-critical Graphs}

Finding forbidden minors of low connectivity now reduces to finding T-critical graphs. This section explores ways of constructing such graphs.

Proposition 4.1 Let $H$ be T-critical with a single terminal a. Let $b$ be a vertex adjacent to a. Then there is a T-critical graph $H^{\prime}$ as a T-minor of $H$ treating both $a, b$ as terminals.

Proof. $H$ is 2-connected and $Y \Delta Y$ reducible without respecting the terminal. Since $H$ is not 1-terminal reducible, it is certainly not 2-terminal reducible (with terminals $a, b)$. By Lemma 3.4, there is a sequence of edge deletions/contractions that reduces $H$ to a 2-terminal critical graph $H^{\prime}$. (The $Y \Delta$ transformation is not needed with only two terminals).

Similarly, we have

Proposition 4.2 Let $H$ be T-critical with two terminals a,b. Suppose $c$ is adjacent to both $a, b$. Then there is a T-critical graph $H^{\prime}$ with three terminals a,b,c obtained from $H$ by a sequence of edge deletions/contractions and $Y \Delta$ transformations.

On the other hand, from a 3-terminal critical graph, we can obtain a 2-terminal critical graph as follows:

Proposition 4.3 Let $H$ be $T$-critical with three terminals a,b,c. Form $H^{\prime}$ from $H$ by adding two new vertices $d, e$ and six new edges ad, ae, bd, be, cd,ce. Declare d,e to be the terminals of $H^{\prime}$. Then there is a T-minor of $H^{\prime}$ that is T-critical.

Proof. $H^{\prime}+\Delta$ is $Y \Delta Y$ reducible by first eliminating $d$, e then following the reductions of $H+\Delta$. Since $a, b, c$ in $H$ each has degree at least two, each has degree at least four in $H^{\prime}$. Also, there are no edges between $a, b, c$ and between $d, e$ in $H^{\prime}$. Hence the only $Y \Delta Y$ transformations that apply to $H^{\prime}$ respecting the terminals are those that apply to $H$, respecting its terminals $a, b, c$. These still hold even after the $Y \Delta Y$ transformations. It is now clear that $H^{\prime}$ is not T-reducible because $a, b, c$ cannot be eliminated. It is easy to see that $H^{\prime}+\Delta$ is 2 -connected. The claim therefore follows by Lemma 3.4 as in Proposition 4.1 . 
Figure 2: $k$-terminal critical graphs with $k=1,2,3$.
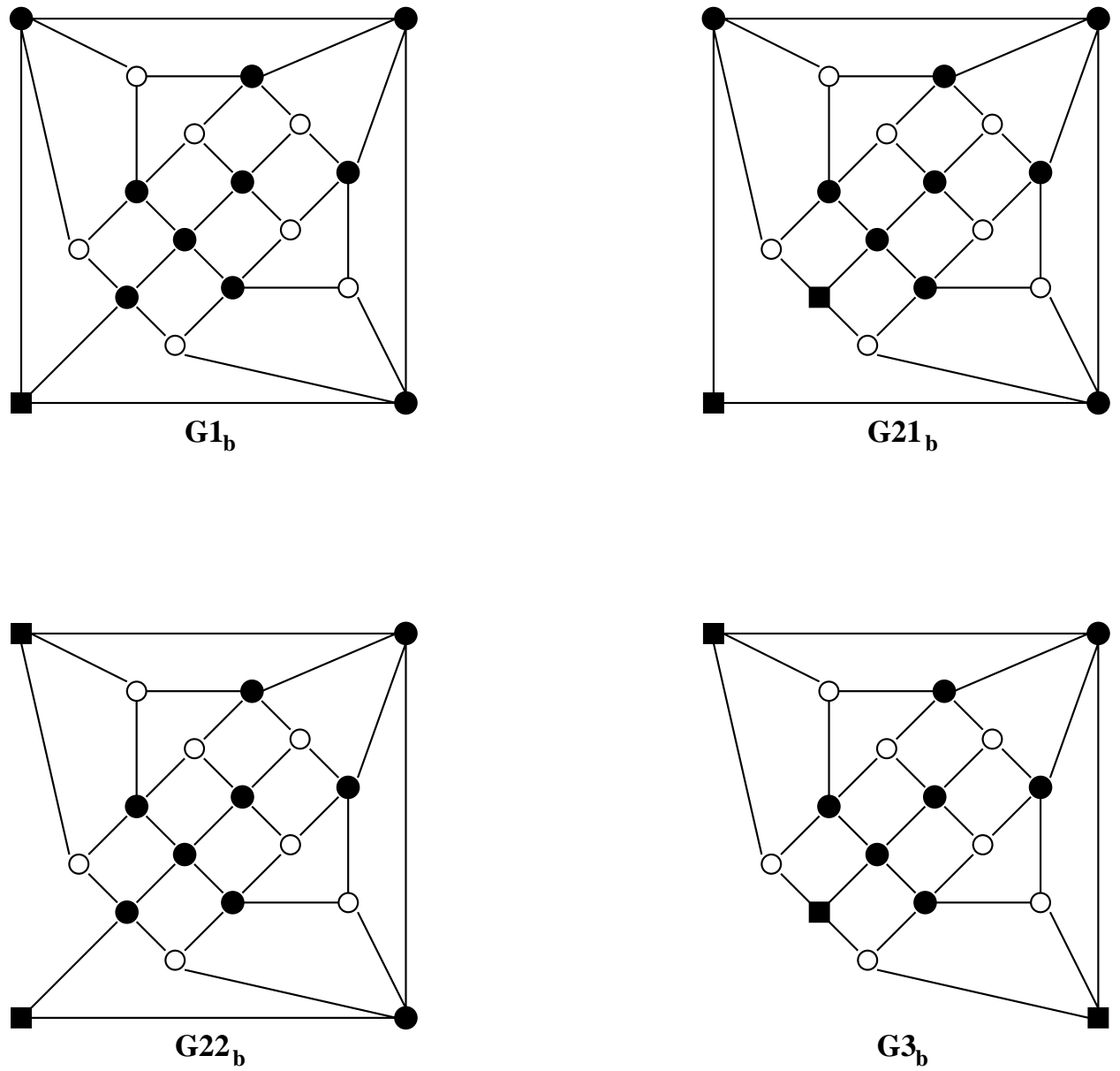

So far we have not yet shown any example of T-critical graphs. Finding such graphs is nontrivial since the conditions are very restrictive. According to Gitler [4], all planar graphs are 3-terminal $Y \Delta Y$ reducible. Thus $k$-terminal critical graphs with $k \leq 3$ are necessarily non-planar. The graph $G_{b}$ in Figure 1, found by Yu [10], leads to an easy proof of the existence of $k$-terminal critical graphs. Consider the following graphs in Figure $2\left(G 1_{b}\right.$ in particular) derived from $G_{b}$. (All four are apex, and terminals are marked by a square rather than a circle; the implicit vertex is not a terminal.) Without respecting the terminal, $G 1_{b}$ is reducible because it is a minor of $G_{b}$; when the terminal is respected, no reductions/transformations can be applied. It is trivial to check the connectivity requirement. By Lemma 3.4, 1-terminal critical graphs exist. In fact, all four are T-critical.

A computer aided search turns up more T-critical graphs. These are derived from $G_{a}$ in Figure 1. Three $Y \Delta Y$ equivalent families of $k$-terminal critical graphs are discovered, one for each of $k=1,2,3$. Three representatives are depicted in Figure 3. 
Figure 3: More $k$-terminal critical graphs with $k=1,2,3$.

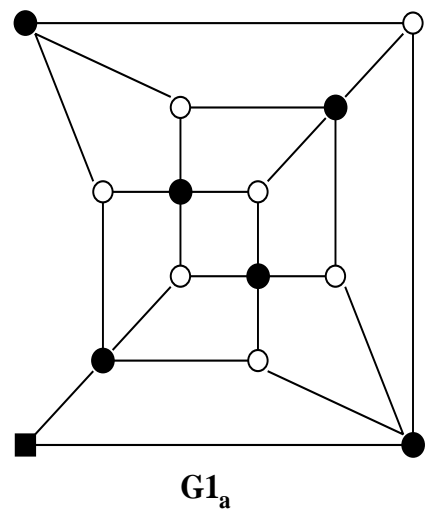

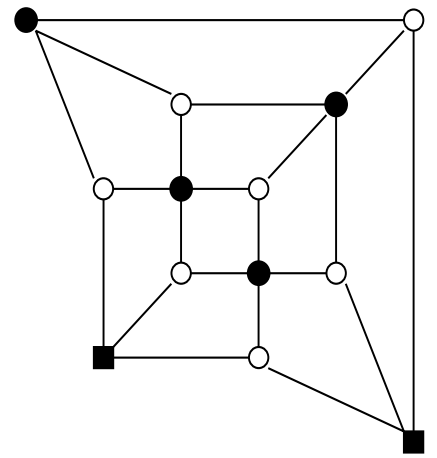

$\mathbf{G}_{\mathrm{a}}$

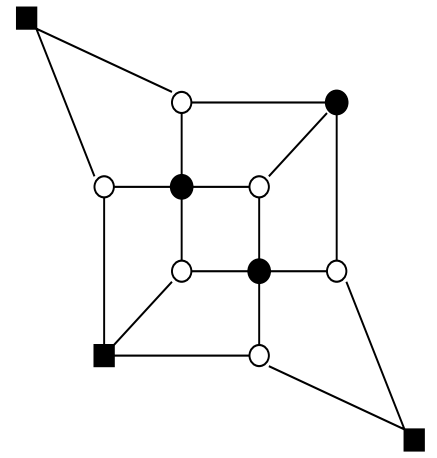

$\mathbf{G 3}_{\mathrm{a}}$

The computer is used to show that each graph in Figure 2 and Figure 3 satisfies the definition of T-criticality. In particular, to verify that one of the graphs (say $G_{0}$ ) is not T-reducible, we run the following program:

- Step 1. Let $L$ be a list of graphs. Initialize $L=\left\{G_{0}\right\}$. Initialize the current size of the list $n=1$. Initialize the current search position $i=0$.

- Step 2. Consider the graph $G_{i}$. If series-parallel reductions are possible, stop; the claim is false.

- Step 3. Find all graphs that can be obtained from $G_{i}$ by a single $Y \Delta$ or $\Delta Y$ transformation, respecting the terminals. For each of these graphs, check if it is T-isomorphic to one already in the list, i.e., the graphs $G_{j}, 0 \leq j<n$. If not, denote the new graph by $G_{n}$, append $G_{n}$ to the list, and increment $n$.

- Step 4. Increment $i$. If $i<n$, go to Step 2; otherwise stop, and the claim is proved.

Testing of T-isomorphism is performed frequently in the above program. We use the Nauty package of B. D. McKay [5,6]. To verify that every T-minor of $G_{0}$ is T-reducible, we can either check by hand, or use trial-and-error with the computer. All graphs that are $Y \Delta Y$ equivalent to $G_{0}$ form a family of T-critical graphs.

It is helpful to classify these graphs by the number of terminals and symmetry structures of the terminals. Specifically, let $S_{k}$ be the symmetric group of order $k \leq 3$. For a $k$-terminal critical graph $G_{0}$ with terminals labeled $1,2, \ldots, k$, let $\Gamma_{k}$ be a subset of $S_{k}$ such that $p \in \Gamma_{k}$ if and only if there is a terminal-preserving automorphism of $G_{0}$ that maps terminal $i$ to $p(i), i=1, \ldots, k$. Obviously $\Gamma_{k}$ is a subgroup of $S_{k}$. T-critical graphs $Y \Delta Y$ equivalent to the seven graphs depicted in Figure 2 and Figure 3 are classified by the number of terminals $k$ and the order of the group $\Gamma_{k}$ as follows:

- There are two families of 1-terminal critical graphs. One has 342288 members, one of which is $G 1_{a}$ in Figure 3. The other has only a single member, which is $G 1_{b}$ in Figure 2. 
Table 1: Number of $k$-terminal critical graphs found, by $\left|\Gamma_{k}\right|$.

\begin{tabular}{lcccc}
\hline \hline & $\left|\Gamma_{k}\right|=1$ & $\left|\Gamma_{k}\right|=2$ & $\left|\Gamma_{k}\right|=3$ & $\left|\Gamma_{k}\right|=6$ \\
\hline$k=1$ & $342288+1$ & & & \\
$k=2$ & $101303+1+1$ & 518 & & \\
$k=3$ & 646 & $61+1$ & 7 & 5 \\
\hline
\end{tabular}

- There are three families of 2-terminal critical graphs. One has 101821 members, one of which is $G 2_{a}$ in Figure 3. 101303 of these satisfy $\left|\Gamma_{2}\right|=1$, whereas the other 518 satisfy $\left|\Gamma_{2}\right|=2$. The other two families each has a single member satisfying $\left|\Gamma_{2}\right|=1$. These are $G 21_{b}$ and $G 22_{b}$ of Figure 2 to be exact.

- There are two families of 3-terminal critical graphs. One has 719 members, one of which is $G 3_{a}$ of Figure 3. 646 of these have $\left|\Gamma_{3}\right|=1,61$ have $\left|\Gamma_{3}\right|=2,7$ have $\left|\Gamma_{3}\right|=3$, and 5 have $\left|\Gamma_{3}\right|=6$. The other family has a single member with $\left|\Gamma_{3}\right|=2$, which is $G 3_{b}$ in Figure 2.

The findings are summarized as Table 1.

We can now compute the total number of minor-minimal $Y \Delta Y$ irreducible graphs with essential cuts of order $k \leq 3$. Suppose $G, G^{\prime}$ are such forbidden minors with cuts $\left(G_{1}, G_{2}\right)$ and $\left(G_{1}^{\prime}, G_{2}^{\prime}\right)$ of order $k$, respectively. Suppose $k$ is minimal of its kind. Denote $C=V\left(G_{1}\right) \cap V\left(G_{2}\right)$ and $C^{\prime}=V\left(G_{1}^{\prime}\right) \cap V\left(G_{2}^{\prime}\right)$. Suppose there is an isomorphism $f$ from $G$ to $G^{\prime}$. Since the cut set is unique, $f$ maps $C$ to $C^{\prime}$. Since $G_{i}-C, i=1,2$ and $G_{i}^{\prime}-C^{\prime}, i=1,2$ are connected, either $f\left(G_{i}-C\right)=G_{i}^{\prime}-C^{\prime}, i=1,2$, or $f\left(G_{1}-C\right)=G_{2}^{\prime}-C^{\prime}$ and $f\left(G_{2}-C\right)=G_{1}^{\prime}-C^{\prime}$. Hence $G_{1}$ is T-isomorphic to $G_{1}^{\prime}$ and $G_{2}$ is T-isomorphic to $G_{2}^{\prime}$, or vice versa. On the other hand, given two $k$-terminal critical graphs $G_{1}, G_{2}$, the number of forbidden minors up to isomorphism that can be constructed by combining them in this manner depends on the group $\Gamma_{k}$ of each. Specifically, there is only one possibility if $k=1$. When $k=2$ there is one possibility if $\left|\Gamma_{2}\right|=2$ is satisfied by one of $G_{i}, i=1,2$, and two possibilities otherwise. Suppose $k=3$. There is only one possibility if $\left|\Gamma_{3}\right|=6$ is satisfied by one of $G_{i}, i=1,2$, or $\left|\Gamma_{3}\right|=3$ for one and $\left|\Gamma_{3}\right|=2$ for the other. There are two possibilities if $\left|\Gamma_{3}\right|=3$ for both, or $\left|\Gamma_{3}\right|=2$ for both, or $\left|\Gamma_{3}\right|=3$ for one and $\left|\Gamma_{3}\right|=1$ for the other. There are three if $\left|\Gamma_{3}\right|=2$ for one and $\left|\Gamma_{3}\right|=1$ for the other. Finally, if both satisfy $\left|\Gamma_{3}\right|=1$, then there are five possibilities if $G_{1}$ is T-isomorphic to $G_{2}$, and six otherwise. Hence we have

Proposition 4.4 Denote the number of $k$-terminal critical graphs $(k \leq 3)$ that satisfy $\left|\Gamma_{k}\right|=l$ by $n_{k l}$. The total number of forbidden minors for $Y \Delta Y$ reducibility with essential cuts of order $k \leq 3$ is

$$
\begin{gathered}
n_{11}\left(n_{11}+1\right) / 2+2 n_{21}\left(n_{21}+1\right) / 2+n_{22}\left(n_{22}+1\right) / 2+n_{21} n_{22} \\
+n_{36}\left(n_{36}+1\right) / 2+n_{36}\left(n_{31}+n_{32}+n_{33}\right)+2 n_{33}\left(n_{33}+1\right) / 2+2 n_{33} n_{31}+n_{33} n_{32}
\end{gathered}
$$




$$
+2 n_{32}\left(n_{32}+1\right) / 2+3 n_{32} n_{31}+6 n_{31}\left(n_{31}-1\right) / 2+5 n_{31} .
$$

Combining the above proposition with the newly discovered $k$-terminal critical graphs, we now know of 68897913659 minor-minimal $Y \Delta Y$ irreducible graphs, including the known 57587 that are internally 4-connected.

Theorem 4.1 There are at least 68897913659 minor-minimal $Y \Delta Y$ irreducible graphs, all of which fit in $20 Y \Delta Y$ equivalent families.

Proof. The family of 719 3-terminal critical graphs has members with $\left|\Gamma_{3}\right|=6$. Hence forbidden minors formed by combining two graphs, both in this family, are all $Y \Delta Y$ equivalent. Similarly, forbidden minors formed by combining a graph in this family with $G 3_{b}$ of Figure 2 are also $Y \Delta Y$ equivalent. There are two ways of combining $G 3_{b}$ with itself. Hence there are four $Y \Delta Y$ equivalent families with minimum order of essential separation $k=3$. Similarly, there are nine with $k=2$ and three with $k=1$. In addition, there are four known internally 4-connected families. The 68897913659 graphs therefore fit in 20 families.

\section{Conclusions}

We provide a characterization of minor-minimal $Y \Delta Y$ irreducible graphs that admit essential separations of order $k \leq 3$. Such graphs are obtained by combining two graphs of a special type, called T-critical graphs. Examples of T-critical graphs are shown, which leads to the discovery of large quantities of minor-minimal $Y \Delta Y$ irreducible graphs.

Finding other possible forbidden minors seems hard. The concept of $k$-terminal criticality, in particular, is useful only for $k \leq 3$. It is easy to show that if $G$ is 4 -terminal critical, then $G+\Delta$ is isomorphic to $K_{5}$. In addition, there are no $k$-terminal critical graphs for $k>4$. Extensive computer search for additional T-critical graphs has yielded no result. It is an open question whether there are exactly 68897913659 minor-minimal $Y \Delta Y$ irreducible graphs.

Acknowledgement: The author thanks the referees for their valuable comments.

\section{References}

[1] D. Archdeacon, C. J. Colbourn, I. Gitler, and J. S. Provan. Four-terminal reducibility and projective-planar wye-delta-wye-reducible graphs. J. Graph Theory 33 (2000) 83-93.

[2] G. V. Epifanov. Reduction of a plane graph to an edge by a star-triangle transformation. Soviet Math. Doklady 166 (1966) 13-17.

[3] T. A. Feo and J. S. Provan. Delta-wye transformations and the efficient reduction of two-terminal planar graphs. Operations Research 41 (1993) 572-582. 
[4] I. Gitler. Delta-Wye-Delta Transformations-Algorithms and Applications. Ph.D. Thesis, University of Waterloo, 1991.

[5] B. D. McKay. Practical graph isomorphism. Congressus Numerantium 30 (1981) $45-87$.

[6] B. D. McKay. Nauty User's Guide (Version 1.5), Technical Report TR-CS-90-02. (Department of Computer Science, Australian National University, 1990)

[7] N. Robertson and P. D. Seymour. Graph minors XX: Wagner's Conjecture. preprint, 1988.

[8] K. Truemper. On the delta-wye reduction for planar graphs. J. Graph Theory 13 (1989) 141-148.

[9] K. Truemper. Matroid Decomposition. Academic Press, Inc. Boston, 1992.

[10] Y. Yu. Forbidden minors for wye-delta-wye reducibility. J. Graph Theory 47 (2004) 317-321. 